\title{
Shifting to Remotely Delivered Mental Health Care: Quality Improvement in the COVID-19 Pandemic
}

\author{
Patrick Daigle and Abraham Rudnick *(1) \\ Department of Psychiatry and School of Occupational Therapy, Nova Scotia Operational Stress Injury Clinic, \\ Dalhousie University, Halifax, NS B3H 4R2, Canada; patrick.daigle@nshealth.ca \\ * Correspondence: harudnick@hotmail.com
}

Received: 31 August 2020; Accepted: 22 September 2020; Published: 22 September 2020

check for updates

\begin{abstract}
This paper presents an organizational (ambulatory) case study of shifting mental health care from in-person to remote service delivery due to the current (COVID-19) pandemic as a rapid quality improvement initiative. Remotely delivered mental health care, particularly using synchronous video and phone, has been shown to be cost-effective, especially for rural service users. Our provincial specialized mental health clinic rapidly shifted to such remote delivery during the current pandemic. We report on processes and outputs of this rapid quality improvement initiative, which serves a purpose beyond pandemic circumstances, such as improving access to such specialized mental health care for rural and other service users at any time. In conclusion, shifting specialized mental health care from in-person to remotely delivered services as much as possible could be beneficial beyond the current pandemic. More research is needed to optimize the implementation of such a shift.
\end{abstract}

Keywords: pandemic; quality improvement; remote delivery; rural; specialized mental health care

\section{Introduction}

Approximately a fifth of the general population in developed countries such as Canada experience at least one disruptive mental disorder in their lifetime [1], and even more experience mental health challenges in many developing countries [2]. Many people live in urban environments, and hence may benefit from mental health services, such as in-person mental health care, recognizing that due to social disparities and other factors in most countries such care is often suboptimal [3]. Many other people live in rural and remote environments and hence have less access to mental health care and related services; digital services show promise to improve such access [4]. During the current (COVID-19) pandemic, even urban populations cannot easily or at all access in-person mental health services. Hence, digital (video and other, such as phone) remotely delivered mental health services may be particularly helpful both during pandemic times as well as more generally such as for people who live rurally or remotely [5]. Although remotely delivered mental health care and related services such as self-help have been shown to be effective as well as cost-effective, such as internet-delivered psychotherapy [6] and tele-psychiatry [7], respectively, they have not been widely implemented within and across many jurisdictions [8], partly as startup resourcing is needed for such change (recognizing that there may be no known disadvantage compared to in-person care for service users who have access to relevant technology and feel comfortable with it). Arguably, it is unethical to not provide remotely delivered mental health services as needed, such as to rural and remote populations in general [9] and to anyone in need during pandemic [10]. Indeed, the current pandemic is an opportunity to shift to such remotely delivered services as much as needed. The rest of this brief paper reports what processes and outputs occurred when our specialized mental health care clinic shifted during the current pandemic from in-person to remotely delivered mental health services. We conclude with suggestions that may 
assist other mental health services and policy makers and that would require rigorous evaluation and research.

\section{Shifting to Remotely Delivered Mental Health Care}

Our clinic is the Nova Scotia Operational Stress Injury Clinic (NSOSIC). It is a Canadian federally funded provincial service that provides specialized mental health care for eligible members and Veterans of the Canadian Armed Forces (CAF) and some North Atlantic Treaty Organization (NATO) Veterans as well as for eligible members and Veterans of the Royal Canadian Mounted Police (RCMP). It is part of a national network of such Operational Stress Injury Clinics across Canadian provinces. The NSOSIC is also part of the Nova Scotia Health Authority (NSHA), is affiliated with Dalhousie University's Faculties of Health and Medicine, and is led by this paper's two authors. It has a main site (in Dartmouth, which is in Nova Scotia's central zone that is urban and suburban) and a pilot satellite site (in Cape Breton, which is in Nova Scotia's Eastern zone that is semi-urban and rural). The NSOSIC's services involve outpatient mental health assessments and biopsychosocial interventions, such as evidence-based/informed individual and group trauma-focused and other psychotherapies, medications, occupational therapy such as for cognitive remediation, and primary care, delivered by an inter-professional team composed of nurses, occupational therapists, primary care providers, psychiatrists, psychologists, and social workers. It was established in 2015 and provided since then some remotely delivered mental health care (primarily tele-psychiatry to rurally residing service users) but mostly in-person (in-office more than outreach) services; those remotely delivered services were primarily provided by the NSOSIC's physicians (who are located in the main site) for service providers residing in Cape Breton who could not or would not fly or drive long distance to the main site; until the current pandemic, some resistance to remotely delivered health care was expressed by some of the NSOSIC's psychotherapists (as noted below), as well as by some of the other providers at the NSOSIC and by some service users and their families as well as by some other third parties such as by some referral sources.

For the first few months of the current pandemic, the NSOSIC could not provide in-person services and had to consider shifting all of its services to remote delivery (since then, the NSOSIC has been providing some in-person services although much less so than remotely delivered services; Figure 1). After a couple of weeks of service shut down, similar to other elective specialized outpatient services of the NSHA, the NSOSIC reopened, initially with only remotely delivered services. As tele-psychiatry had been provided (to a much lesser extent) before the current pandemic, including by secure video software (Medeo), shifting all of its psychiatry services to remote delivery was rapid and fairly easy and simple, including adding an alternative secure video software (Zoom Healthcare, approved by the NSHA) in case the Medeo video software did not work well for some service users (and vice versa). Other individual services were initially more challenging to shift to remote delivery, for historical and other reasons, e.g., some psychotherapists were previously not comfortable with providing such confidential care from home to home, although within a few weeks that challenge was resolved by means of the clinic's leadership clarification of safeguards and the psychotherapists' recognition of the need to provide care to service users; clinicians also had to set up secure internet access at their homes with the clinic's hardware and software to ensure they had access to all necessary resources such as the NSOSIC's electronic health record, and they were provided formal orientation (by NSHA) to remotely delivered services. Group interventions were delayed for longer due to more complex confidentiality challenges, which were resolved within a few months by the NSHA for all of its mental health services. Occupational therapy proceeded rapidly (within a few weeks) with the shift to remote delivery, as did primary care (with exceptions when a physical examination was required). Sessions length varied according to care plan and type of intervention, e.g., half an hour for psychiatry follow up and $\sim$ an hour for psychotherapy (no different than in person care). As the NSOSIC uses an electronic health record and digital forms, documentation proceeded seamlessly, with e-signatures and their digital equivalents approved by the NSHA leadership. 
To illustrate the rapid shift to remotely delivered clinical services by internet-based (synchronous) video and phone at the NSOSIC soon after the current pandemic started, we highlight here a couple of process indicators in support of that (recognizing that, in health care, process quality is distinct from content quality, which has to be addressed with standardized clinical measures that cannot be reported here due to proprietary and other considerations). We use the term rapid to mean weeks to very few (a couple of) months, as typically change in health care is much slower, i.e., in the order of years. First, the ratio of in-person to remotely delivered services changed extremely, as, compared to 2019, when more than $95 \%$ of mental health care was delivered in-person, in May-July 2020, more than $90 \%$ of mental health care was delivered remotely (Figure 1); the first four months of 2020 are confounded by a mix of pre-pandemic data and start-of-pandemic constraints (of initial closure of non-urgent services such as the NSOSIC), hence their data are not addressed here (also note that the number of clinicians is addressed in Figure 1; this number includes an occupational therapist, a psychiatrist and the psychotherapists at the NSOSIC and varies somewhat from week to week due to their leaves). Second, deviations from planned care such as psychotherapy protocols reduced soon after full implementation of remote delivery of services by the NSOSIC, as monitored (by clinician self-report) during 3 months: May-July 2020 (Figure 2); this suggests that both the clinicians and the service users gradually, yet fairly rapidly, felt more skilled and comfortable with remotely delivered mental health services (assessments and care), although a few service users had continued difficulty with not meeting their psychotherapist in person (hence within a few weeks they were shifted back to in person care, using pandemic safeguards such as physical distancing and protective masks). The number of referrals to the NSOSIC decreased at the start of the current pandemic, as expected due to the initial closure of provincial non-urgent services such as the NSOSIC; that soon (within a couple of months) increased-approximately back to the regular number-and wait times for clinical care did not lengthen once the NSOSIC reopened soon after the start of the current pandemic. Although the current pandemic did not considerably change the clinical needs of the service users (largely as addressing social isolation is often part of the clinical focus of the NSOSIC), a mass shooting in Nova Scotia in April 2020 added stress and a few more referrals, particularly in relation to some RCMP officers who were directly or indirectly involved in that shooting. There were a few internet breakdowns in the remote delivery technology, mainly with rural service users, for which phone contact was used as a backup (allowing follow up more than other interventions in real time).

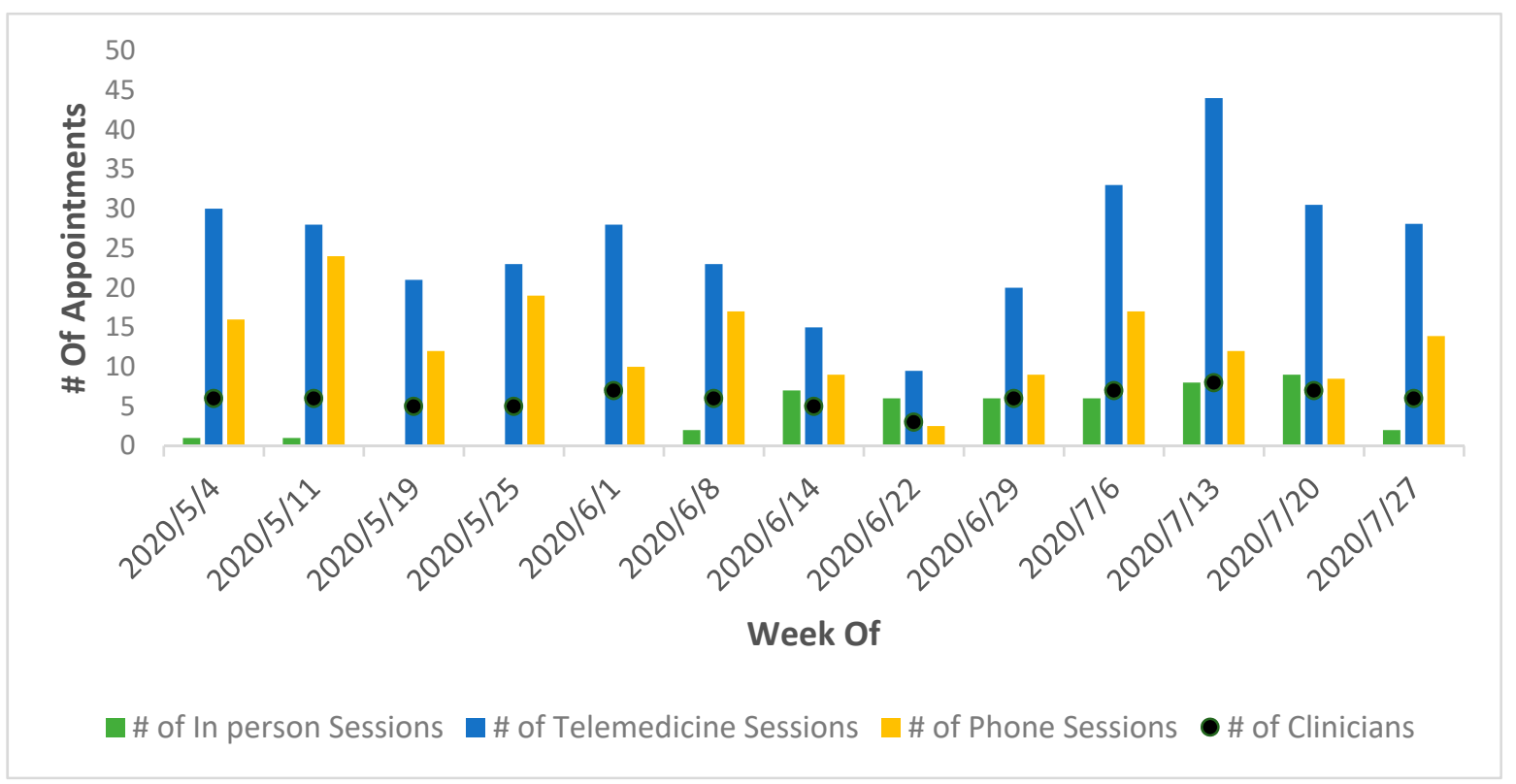

Figure 1. Remotely delivered vs. in-person mental health care at the Nova Scotia Operational Stress Injury Clinic (NSOSIC) (appointments/sessions per week). 


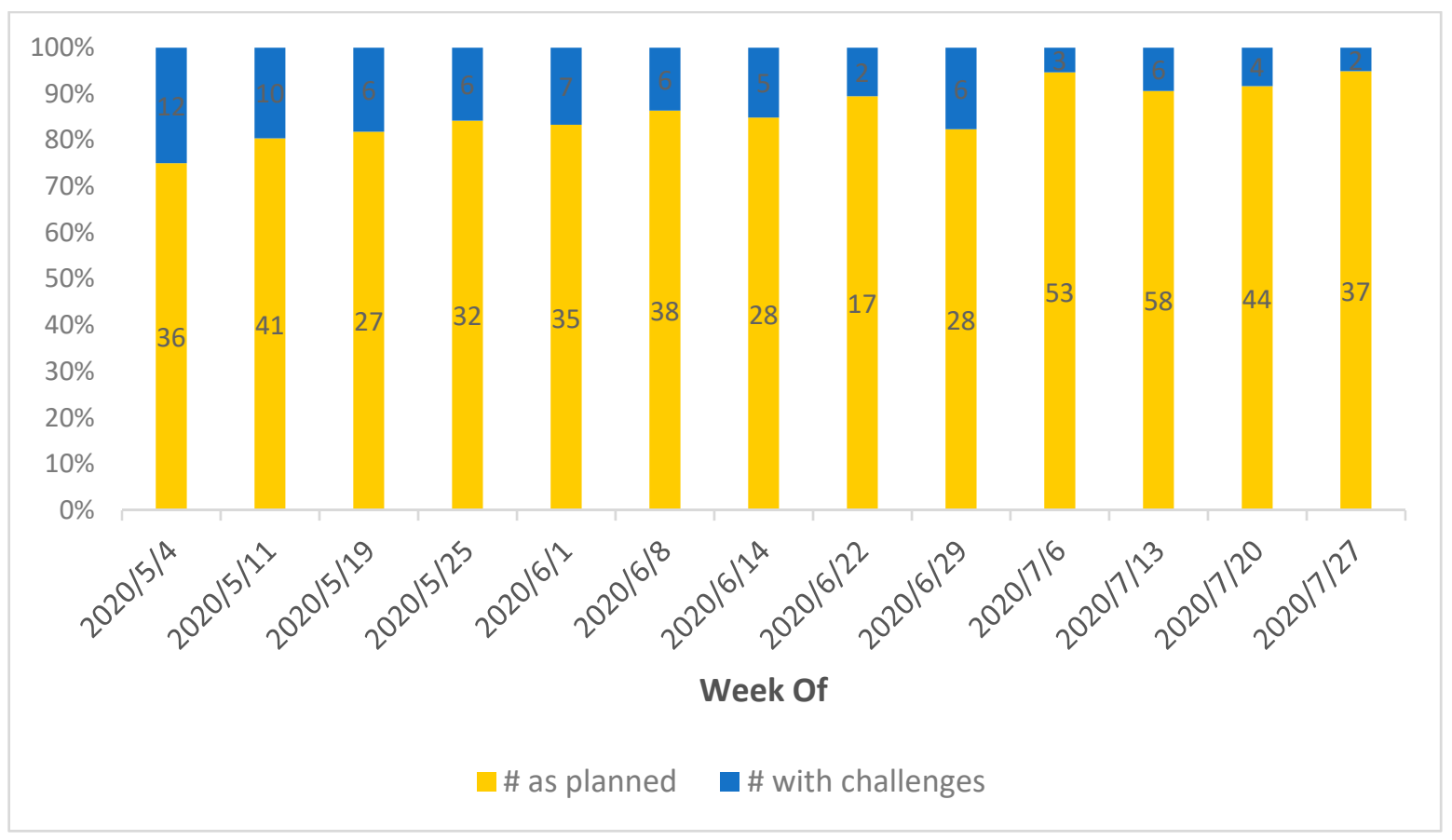

Figure 2. Deviations from planned care at the NSOSIC (appointments/sessions per week).

\section{Conclusions}

Remotely delivered mental health care and related services can be effective and likely cost-effective, as shown in published research. Such services can also be implemented rapidly, as illustrated in this quality improvement report about the NSOSIC's recent shift to remotely delivered (specialized and integrated) services in the current pandemic. As part of that, evidence-based/informed mental health assessments and interventions can be provided, with little deviation from planned care after a relatively short period - a few weeks to a couple of months - of transition from (mostly) in-person to (mostly) remotely delivered services. Beyond the pandemic circumstances, such a shift can benefit people with mental health challenges who live rurally or remotely, so that their access to mental health services is not compromised because of their distant physical location, and indirect costs of their care may be reduced due to eliminating the need for them to drive long distances (or fly) to and from the physical location of the service providers. Admittedly, universal broadband (or equivalent, such as satellite) internet access is needed, which is in progress in Canada and elsewhere, as well as sufficiently user friendly hardware and software for all to use at an affordable cost. Our report is limited in that it reports a local (process) quality improvement initiative rather than a research project. Hence, generalized conclusions have to be qualified and well controlled research is needed to address processes, outcomes and their predictors in relation to shifting to remotely delivered mental health care. Nevertheless, our findings are promising for such a shift and suggest the need to proceed with such change in practice and policy, e.g., facilitating universal access to digital technology (hardware, software, and broadband/satellite internet). Hopefully, cross-sector collaborations will advance and uphold such access and person-centered use for the benefit of the large number of people with mental health challenges across the world.

Author Contributions: Both authors contributed to the planning, data collection, data analysis/interpretation, and writing of this report. All authors have read and agreed to the published version of the manuscript.

Funding: This report received no external funding.

Conflicts of Interest: The authors declare no conflict of interest. 


\section{References}

1. Richter, D.; Wall, A.; Bruen, A.; Whittington, R. Is the Global Prevalence Rate of Adult Mental Illness Increasing? Systematic Review and Meta-Analysis. Acta Psychiatr. Scand. 2019, 140, 393-407. [CrossRef] [PubMed]

2. Charlson, F.; van Ommeren, M.; Flaxman, A.; Cornett, J.; Whiteford, H.; Saxena, S. New WHO Prevalence Estimates of Mental Disorders in Conflict Settings: A Systematic Review and Meta-Analysis. Lancet 2019, 394, 240-248. [CrossRef]

3. Kilbourne, A.M.; Beck, K.; Spaeth-Rublee, B.; Ramanuj, P.; O’Brien, R.W.; Tomoyasu, N.; Pincus, H.A. Measuring and Improving the Quality of Mental Health Care: A Global Perspective. World Psychiatry 2018, 17, 30-38. [CrossRef] [PubMed]

4. Rojas, G.; Martinez, V.; Martinez, P.; Franco, P.; Jimenez-Molina, A. Improving Mental Health Care in Developing Countries Through Digital Technologies: A Mini Narrative Review of the Chilean Case. Front. Public Health 2019, 7, 391. [CrossRef] [PubMed]

5. Rudnick, A. Remote Psychosocial Rehabilitation (rPSR): A Broad View. J. Psychosoc. Rehabil. Ment. Health 2020, 7, 119-120. [CrossRef] [PubMed]

6. Wagner, B.; Horn, A.B.; Maercker, A. Internet-Based versus Face-to-Face Cognitive Behavioral Intervention for Depression: A Randomized Controlled Non-Inferiority Trial. J. Affect. Disord. 2013, 152, 113-121. [CrossRef] [PubMed]

7. O’Reilly, R.; Bishop, J.; Maddox, K.; Hutchinson, L.; Fisman, M.; Takhar, J. Is Telepsychiatry Equivalent to Face-to-Face Psychiatry? Results from a Randomized Controlled Equivalence Trial. Psychiatr. Serv. 2007, 58, 836-843. [CrossRef] [PubMed]

8. Chakrabarti, S.; Shah, R. Telepsychiatry in the Developing World: Whither Promised Joy? Indian J. Soc. Psychiatry 2016, 32, 273-280. [CrossRef]

9. Reglitz, M.; Rudnick, A. Internet Access as a Right for Realizing the Human Right to Adequate Mental (and Other) Health Care. Int. J. Ment. Health 2020, 49, 97-103. [CrossRef]

10. Moreno, C.; Wykes, T.; Galderisi, S.; Nordentoft, M.; Crossley, N.; Jones, N.; Cannon, M.; Correll, C.U.; Byrne, L.; Carr, S.; et al. How Mental Health Care Should Change as a Consequence of the COVID-19 Pandemic. Lancet Psychiatry 2020, 7, 813-824. [CrossRef] 